\title{
Susceptibility-Weighted Imaging in Pediatric Arterial Ischemic Stroke: A Valuable Alternative for the Noninvasive Evaluation of Altered Cerebral Hemodynamics
}

\author{
R.M. Polan, ${ }^{\mathbb{D}}$ A. Poretti, T.A.G.M. Huisman, and T. Bosemani
}

\begin{abstract}
BACKGROUND AND PURPOSE: SWI provides information about blood oxygenation levels in intracranial vessels. Prior reports have shown that SWI focusing on venous drainage can provide noninvasive information about the degree of brain perfusion in pediatric arterial ischemic stroke. We aimed to evaluate the influence of the SWI venous signal pattern in predicting stroke evolution and the development of malignant edema in a large cohort of children with arterial ischemic stroke.
\end{abstract}

MATERIALS AND METHODS: A semiquantitative analysis of venous signal intensity on SWI and diffusion characteristics on DTI was performed in 16 vascular territories. The mismatch between areas with SWI-hypointense venous signal and restricted diffusion was correlated with stroke progression on follow-up. SWI-hyperintense signal was correlated with the development of malignant edema.

RESULTS: We included 24 children with a confirmed diagnosis of pediatric arterial ischemic stroke. Follow-up images were available for 14/24 children. MCA stroke progression on follow-up was observed in 5/6 children, with 2/8 children without mismatch between areas of initial SWI hypointense venous signal and areas of restricted diffusion on DTI. This mismatch showed a statistically significant association $(P=.03)$ for infarct progression. Postischemic malignant edema developed in 2/10 children with and 0/14 children without SWI-hyperintense venous signal on initial SWI $(P=.07)$.

CONCLUSIONS: SWI-DTI mismatch predicts stroke progression in pediatric arterial ischemic stroke. SWI-hyperintense signal is not useful for predicting the development of malignant edema. SWI should be routinely added to the neuroimaging diagnostic protocol of pediatric arterial ischemic stroke.

ABBREVIATIONS: $\mathrm{ACA}=$ anterior cerebral artery; $\mathrm{AIS}=$ arterial ischemic stroke; $\mathrm{ASL}=$ arterial spin-labeling; $\mathrm{CMRO}_{2}=$ cerebral metabolic rate of oxygen; $\mathrm{mIP}=$ minimum intensity projection; OEF = oxygen extraction fraction; PAIS = pediatric arterial ischemic stroke; PSI = prediagnostic symptomatic interval

A cute arterial ischemic stroke (AIS) affects 2-5/100,000 children every year and is associated with high mortality and morbidity. ${ }^{1}$ The mortality rate is estimated at $5 \%-13 \%$, and moderate-to-severe neurologic deficits or epilepsy occur in $>50 \%$ of children after AIS.,3 The Chest and American Heart Association guidelines support the use of anticoagulation in acute pediatric arterial ischemic stroke (PAIS) despite of the absence of largescale clinical trials. ${ }^{4,5}$ Antithrombotic therapy aims to prevent early propagation of the thrombus, inhibit the formation of new

Received September 9, 2014; accepted after revision October 13.

From the Section of Pediatric Neuroradiology, Division of Pediatric Radiology, Russell H. Morgan Department of Radiology and Radiological Sciences, The Johns Hopkins University School of Medicine, Baltimore, Maryland.

Please address correspondence to Thangamadhan Bosemani, MD, Section of Pediatric Neuroradiology, Division of Pediatric Radiology, Russell H. Morgan Department of Radiology and Radiological Science, The Johns Hopkins University School of Medicine, Charlotte R. Bloomberg Children's Center, Sheikh Zayed Tower, Room 4173, 1800 Orleans St, Baltimore, MD 21287-0842; e-mail: tbosema1@jhmi.edu 三 Indicates article with supplemental on-line tables.

http://dx.doi.org/10.3174/ajnr.A4187 thrombus, and promote early recanalization to save hypoperfused tissue at risk of irreversible ischemic infarction. However, the diagnosis of PAIS should be made first, and tissue at risk for infarction should be detected. The diagnosis of PAIS is frequently delayed or missed. ${ }^{6}$ DWI/DTI is a highly sensitive MR imaging sequence in detecting early ischemic regions and is the diagnostic criterion standard for imaging acute PAIS. ${ }^{7}$ Neuroimaging techniques that allow early, reliable, noninvasive identification of potentially salvageable hypoperfused brain tissue-the so called ischemic penumbra-are imperative to guide treatment.

SWI is a high-spatial-resolution, gradient-echo MR imaging sequence that accentuates the magnetic properties of various substances such as blood, blood products, nonheme iron, and calcification. ${ }^{8}$ In addition, SWI accentuates magnetic susceptibility differences between deoxygenated hemoglobin in the vessels and adjacent oxygenated tissues. A few previous reports have shown that SWI-hypointense signals in veins draining hypoperfused brain areas provide indirect evaluation of critically perfused tissue by focusing on venous drainage. ${ }^{9-12}$ In addition, SWI-hyperin- 
tense signal was reported to detect regions of hyperperfusion and to be associated with an increased risk of developing postischemic malignant edema. ${ }^{13}$ SWI may consequently serve as a valuable alternative sequence to evaluate the hemodynamics of brain tissue in PAIS.

The aims of this retrospective study were to evaluate the potential of acute SWI to identify potentially salvageable brain tissue and to predict the development of postischemic malignant edema in the largest cohort of PAIS reported so far, to our knowledge. We hypothesized that hypointense venous signal on acute SWI may identify brain tissue at risk of infarction progression by focusing on venous drainage and that the presence of SWI-hyperintense venous signal may predict the development of postischemic malignant edema.

\section{MATERIALS AND METHODS}

This retrospective study was approved by our institutional review board.

\section{Patients}

The inclusion criteria for this study were the following: 1) a confirmed diagnosis of PAIS (clinical and acute neuroimaging findings including DWI/DTI); 2) the availability of at least $1 \mathrm{MR}$ imaging study of diagnostic quality including at least 3D-T1WI, axial T2WI, FLAIR images, DWI/DTI, and SWI; 3) a prediagnostic symptomatic interval (PSI) of $<8$ days; and 4 ) age at MR imaging of 18 years or younger. Children with hemorrhagic or metabolic stroke and children with acute outside neuroimaging who were transferred to our hospital were excluded from this study. Data from eligible patients were obtained through an electronic search of our pediatric neuroradiology data base covering January 1, 2009, through June 1, 2014.

Clinical histories of patients were reviewed for stroke etiology, symptoms, and clinical findings on admission; PSI; time between diagnosis and neuroimaging follow-up; development of postischemic malignant edema; and acute thrombolytic therapy. PSI was defined as the time interval between onset of symptoms and the neuroimaging diagnosis of PAIS. The presence of a postischemic malignant edema was defined by the presence of neurologic deterioration within 48-72 hours after acute PAIS and uncal or transtentorial herniation on neuroimaging and/or the need for neurosurgical decompression.

\section{Image Acquisition}

All MR imaging studies were acquired on a $1.5 \mathrm{~T}$ clinical MR imaging scanner (Avanto; Siemens, Erlangen, Germany) by using our standard departmental protocol for PAIS, including 3D isotropic T1WI, axial T2WI, FLAIR images, DTI, SWI, arterial spinlabeling (ASL), and MRA.

A single-shot spin-echo echo-planar imaging axial DTI sequence with diffusion gradients along 20 noncollinear directions was performed. An effective high b-value of $1000 \mathrm{~s} / \mathrm{mm}^{2}$ was used for each of the 20 diffusion-encoding directions. An additional measurement without diffusion-weighting $\left(b=0 \mathrm{~s} / \mathrm{mm}^{2}\right)$ was also performed. Acquisition parameters were the following: $\mathrm{TR}=$ $7100 \mathrm{~ms}, \mathrm{TE}=84 \mathrm{~ms}$, section thickness $=2.5 \mathrm{~mm}, \mathrm{FOV}=240 \times$ $240 \mathrm{~mm}$, and matrix size $=192 \times 192$. An integrated parallel acquisition technique $=2$ with generalized autocalibrating partially parallel acquisition reconstruction was used. Diffusion trace and ADC maps were automatically calculated by the vendor-specific software on the MR imaging scanners.

For the SWI sequence, we used the following parameters: $\mathrm{TR}=48 \mathrm{~ms}, \mathrm{TE}=40 \mathrm{~ms}$, flip angle $=15^{\circ}$, bandwidth $=80 \mathrm{kHz}$, section thickness $=1.2 \mathrm{~mm}$ with 128 sections per slab, FOV $=$ $146 \times 180 \mathrm{~mm}$, and matrix size $=256 \times 512$. Integrated parallel acquisition technique factor 2 was used. Minimum intensity projection (mIP) images were automatically reconstructed by the vendor-specific software on the MR imaging scanners. An effective $\mathrm{mIP}$ thickness of $8 \mathrm{~mm}$ was used for neonates, and $16 \mathrm{~mm}$, for older patients. The smaller effective mIP thickness of $8 \mathrm{~mm}$ was used in neonates to limit partial volume effects due to small brain size and any subsequent anatomic misregistration of vessels that might masquerade as pathology.

\section{Image Analysis}

Analysis of all images was performed in consensus by R.M.P., a medical student, A.P., a pediatric neurologist with vast experience in pediatric neuroimaging research, and T.B., a pediatric neuroradiology attending physician. For each cerebral hemisphere, we defined the following vascular territories: anterior cerebral artery (ACA) territory, posterior cerebral artery territory, and 6 cortical regions of the MCA territory (M1-3 at the level of the basal ganglia and M4-6 at the level rostral to the ganglionic structures) according to the Alberta Stroke Program Early CT Score. ${ }^{14}$

Diffusion was graded for each arterial territory as normal or restricted on the basis of ADC maps. SWI signal intensity of the sulcal veins for each arterial territory was graded as hyperintense, isointense, mildly hypointense, or markedly hypointense. In addition, SWI signal intensity of the intramedullary veins in the MCA territories was evaluated and graded in a manner similar to the grading of the sulcal veins. MIP SWI were used for the evaluation. Finally, perfusion in each vascular territory was graded as normal, decreased, or increased. Perfusion was evaluated on relative CBF maps for ASL. The final extent of infarction was evaluated on follow-up neuroimaging as the absence or presence of gliosis or encephalomalacia in each vascular territory.

To identify brain tissue at risk of infarction, mismatch was determined between the number of vascular territories showing restricted diffusion and the number of vascular territories showing mildly and markedly SWI-hypointense venous signal. The number of territories with SWI-hypointense venous signal was graded as greater than, equal to, or less than the number of territories with restricted diffusion. In addition, the number of territories with SWI-hypointense venous signal was compared with the number of territories with abnormal (increased or decreased) perfusion.

To identify infarct progression, the number of vascular territories with restricted diffusion on the initial MR imaging study was compared with the number of vascular territories with gliosis or encephalomalacia on the follow-up neuroimaging study. Infarct progression was defined as a higher number of involved territories with gliosis or encephalomalacia on follow-up imaging 
Table 1: SWI, DWI/DTI, and PWI findings in 24 children with AIS

\begin{tabular}{|c|c|c|c|c|c|c|c|c|c|c|}
\hline & & \multicolumn{4}{|c|}{ SWI Findings $(n=24)$} & \multicolumn{2}{|c|}{ DWI/DTI Findings $(n=24)$} & \multicolumn{3}{|c|}{ ASL Findings $(n=7)$} \\
\hline & & Iso & Hyper & Mildly Hypo & Markedly Hypo & Normal & Decreased & Normal & Perfusion $\downarrow$ & Perfusion $\uparrow$ \\
\hline \multirow[t]{2}{*}{ ACA } & $\mathrm{R}$ & 18 & 4 & 1 & 1 & 20 & 4 & 6 & 1 & 0 \\
\hline & L & 16 & 5 & 2 & 1 & 20 & 4 & 7 & 0 & 0 \\
\hline \multicolumn{11}{|l|}{ MCA } \\
\hline \multirow[t]{2}{*}{ M1 } & $\mathrm{R}$ & 16 & 2 & 4 & 2 & 17 & 7 & 7 & 0 & 0 \\
\hline & L & 15 & 4 & 4 & 1 & 17 & 7 & 6 & 1 & 0 \\
\hline \multirow[t]{2}{*}{ M2 } & $\mathrm{R}$ & 14 & 2 & 6 & 2 & 17 & 7 & 6 & 1 & 0 \\
\hline & L & 14 & 2 & 6 & 2 & 15 & 9 & 6 & 1 & 0 \\
\hline \multirow[t]{2}{*}{ M3 } & $\mathrm{R}$ & 18 & 2 & 2 & 2 & 20 & 4 & 6 & 1 & 0 \\
\hline & L & 20 & 2 & 1 & 1 & 16 & 8 & 7 & 0 & 0 \\
\hline \multirow[t]{2}{*}{ M4 } & $R$ & 17 & 2 & 3 & 2 & 16 & 8 & 6 & 1 & 0 \\
\hline & L & 15 & 4 & 3 & 2 & 16 & 8 & 6 & 0 & 1 \\
\hline \multirow[t]{2}{*}{ M5 } & $R$ & 13 & 3 & 6 & 2 & 13 & 11 & 6 & 1 & 0 \\
\hline & L & 13 & 2 & 5 & 4 & 13 & 11 & 5 & 0 & 2 \\
\hline \multirow[t]{2}{*}{ M6 } & $R$ & 18 & 3 & 1 & 2 & 16 & 8 & 6 & 1 & 0 \\
\hline & L & 19 & 2 & 2 & 1 & 18 & 6 & 7 & 0 & 0 \\
\hline \multirow[t]{2}{*}{ IMV } & $R$ & 17 & 0 & 6 & 1 & & & & & \\
\hline & L & 20 & 0 & 2 & 2 & & & & & \\
\hline \multirow[t]{2}{*}{ PCA } & $R$ & 15 & 4 & 3 & 2 & 20 & 4 & 5 & 2 & 0 \\
\hline & L & 12 & 6 & 5 & 1 & 18 & 6 & 4 & 1 & 2 \\
\hline
\end{tabular}

Note:-IMV indicates intramedullary veins; Iso, isointense; Hyper, hyperintense; Hypo, hypointense; $\uparrow$, increased; $\downarrow$, decreased; R, right; L, left; PCA, posterior cerebral artery.

compared with the initial DTI. For each patient, the extent of the infarcted tissue on follow-up imaging was graded as progression or none.

\section{Statistical Analysis}

The Pearson $\chi^{2}$ test was used to compare the ability of the DTISWI hypointense venous signal mismatch to predict stroke progression within the 6 cortical regions of the MCA territory and the ability of SWI-hyperintense venous signal to predict the development of malignant edema. ASPECTS vascular territories were used to better define the extent of MCA stroke on initial imaging and to detect progression on subsequent imaging. Analyses were performed by using STATA software, Version 12.1 (StataCorp, College Station, Texas). Observed differences were considered statistically significant if the $P$ value was $<.05$.

\section{RESULTS}

Twenty-four children (10 girls) were included in our study (Online Table 1). The median age of patients at acute stroke presentation was 4.7 years (range, 2 days to 17 years). Risk factors for PAIS were present in 18 children (75\%). The most common risk factors were congenital heart disease in 5 (21\%) and Moyamoya syndrome in $4(17 \%)$ children. The average PSI was 2 days (range, $0-7$ days). None of the patients had acute thrombolytic therapy.

The results of the semiquantitative evaluation of acute DTI, SWI, and ASL data are shown in Table 1. ASL data were available for 7 children. Follow-up neuroimaging studies were available for 15 children (63\%) and were performed, on average, 47 days after acute neuroimaging (range, 1 day to 8 months). Areas of gliosis or encephalomalacia were seen in 14/24 children, including all vascular territories. In the acute neuroimaging studies, a mismatch between SWI and DWI/DTI was found in 22 patients (92\%). The number of vascular territories with SWI-hypointense venous signal was greater than the number of vascular territories with restricted diffusion in 15 children (63\%) and smaller in 7 children

\begin{tabular}{lccc}
$\begin{array}{l}\text { Table 2: SWI/DWI mismatch in the MCA territories predicts } \\
\text { stroke evolution on follow-up imaging in } 14 \text { children with AIS }\end{array}$ \\
$\begin{array}{cccc}\text { No Stroke Progression } \\
\text { on Follow-Up }\end{array}$ & $\begin{array}{c}\text { Stroke Progression } \\
\text { on Follow-Up }\end{array}$ & Total \\
\hline SWI/DWI & 6 & 2 & 8 \\
\hline SWI = DWI & 1 & 5 & 6 \\
SWI > DWI & 7 & 7 & 14 \\
Total & &
\end{tabular}

Pearson $\chi^{2}$ test $=0.03$.

(29\%). In 2 children (8\%), the vascular territories with SWI-hypointense venous signal and restricted diffusion matched. A mismatch between SWI and ASL was found in 6/7 patients (86\%). The vascular territories with SWI-hypointense venous signal were greater than the vascular territories with abnormal perfusion in 3 children and smaller in 3 children. In 1 child, the vascular territories with SWI-hypointense venous signal and reduced perfusion matched perfectly.

The results of the semiquantitative evaluation of acute DTI, SWI, and ASL data of 14 children with a stroke within at least 1 of the 6 cortical regions of the right or left MCA territory are shown in On-line Table 2. Follow-up images were available for all 14 children included in the statistical analysis. A mismatch between SWI hypointense veins with vascular territories greater than those on DTI was found in 6 children (Table 2), of which 5 children showed infarct progression. This mismatch between SWI and DTI was significantly associated with stroke progression on follow-up imaging $(P=.03)$ (Figs 1 and 2).

Malignant edema developed in 2 of 24 children ( $8 \%$ ). In both patients, brain edema was diffuse and SWI-hyperintense venous signal involved the right ACA and MCA territories in the first patient (Fig 3) and the bilateral MCA and left posterior cerebral artery territories in the other. In both patients, ASL data were not available. SWI-hyperintense venous signal was found in 8 children with AIS who did not develop postischemic malignant edema. The presence of SWI-hyperintense venous signal was not significantly associated with the development of postischemic malignant edema $(P=.07)$ (Table 3$)$. 

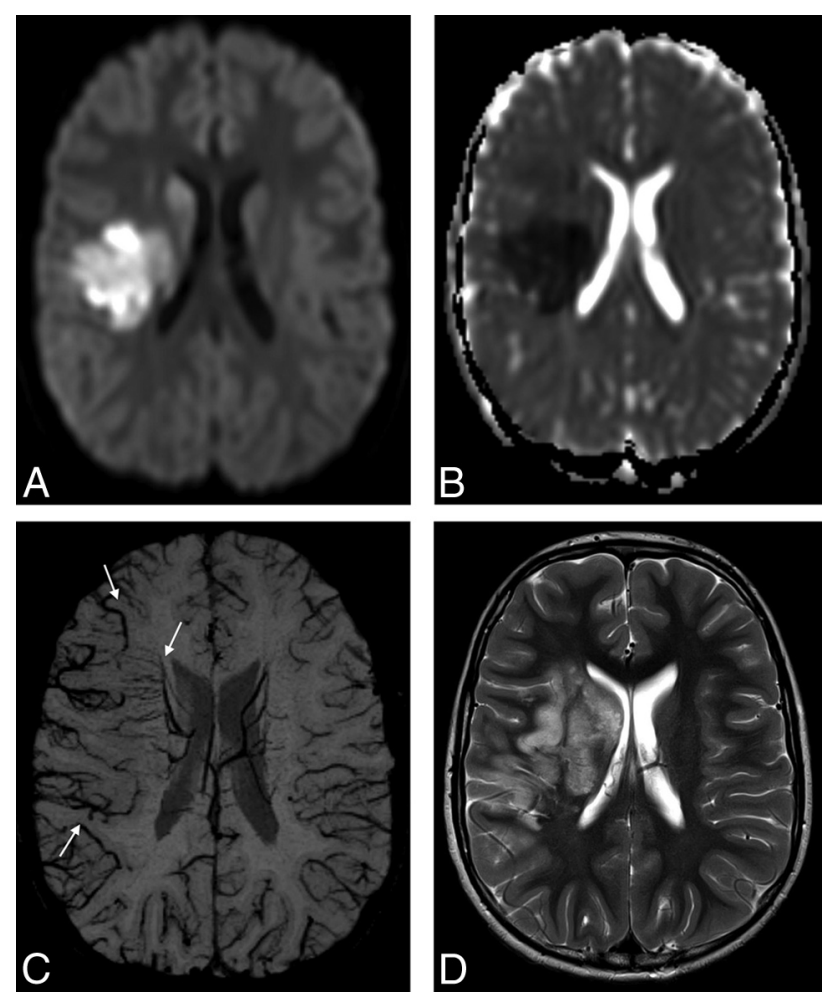

FIG 1. An 8-year-old boy with elevated lipoprotein A and AIS involving the right MCA territory. Trace of diffusion $(A)$ and ADC $(B)$ maps show areas of restricted diffusion in the right basal ganglia and part of the subcortical white matter and cortical gray matter in the right MCA territory, representing acute ischemia. C, mIP-SWI map shows markedly hypointense sulcal and intramedullary veins within the larger right MCA territory (arrows). D, Follow-up axial T2-weighted image 6 days after AIS shows hyperintense signal in the infarcted brain tissue that extends beyond the vascular territories with restricted diffusion and matches the area with SWI-hypointense veins on acute neuroimaging.

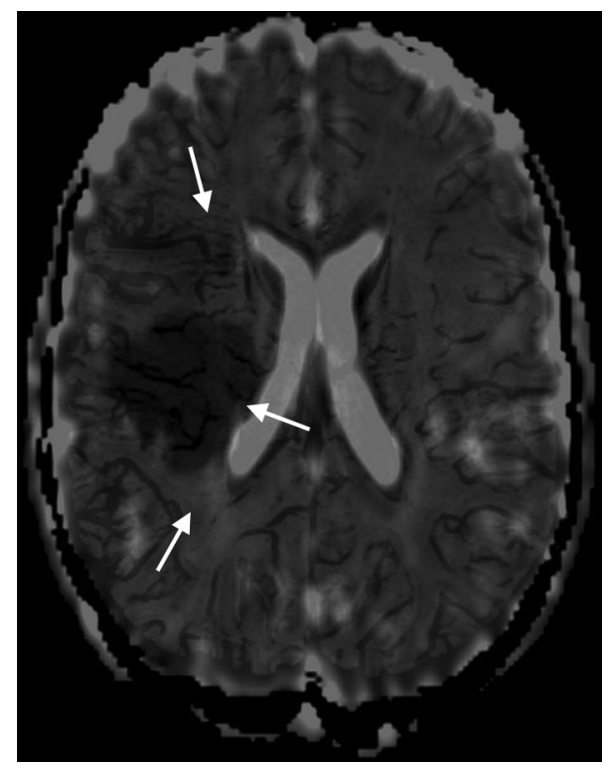

FIG 2. Fused axial ADC and mIP-SWI map images for the same child as in Fig 1 show that markedly hypointense sulcal and intramedullary veins on SWI are draining an area that extends beyond the region of restricted diffusion on the ADC map in the right MCA territory.
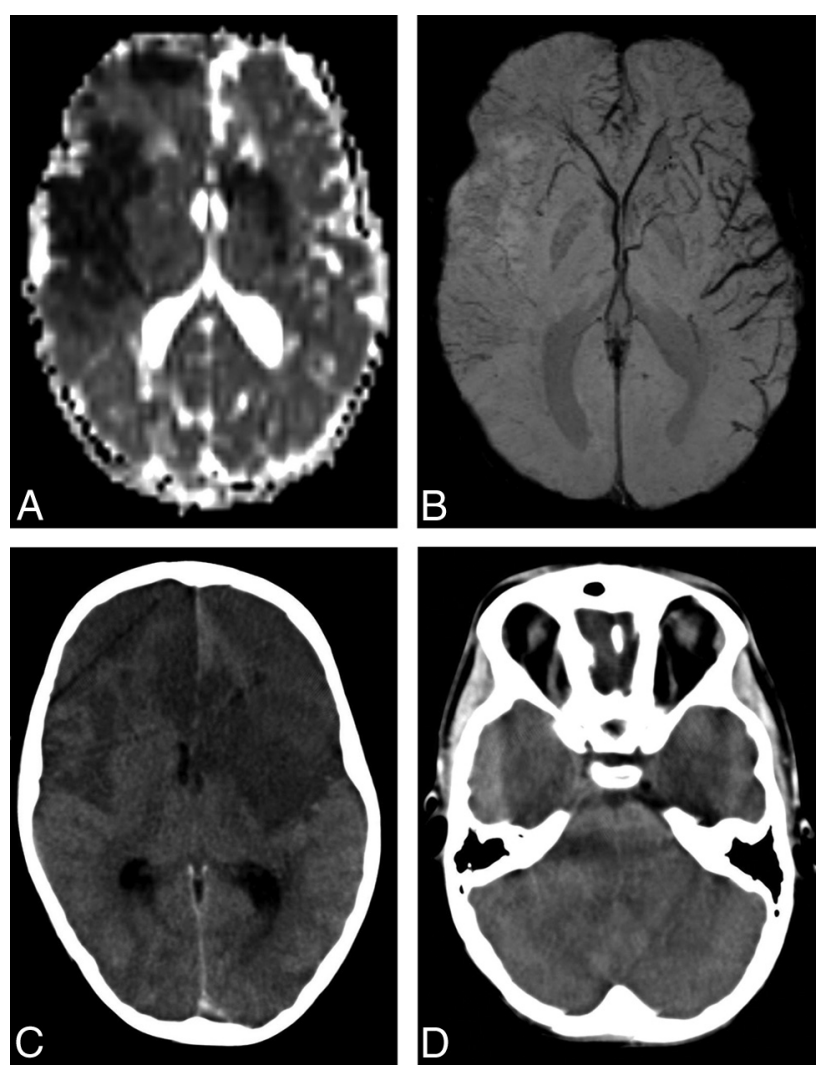

FIG 3. A 7-year-old boy with AIS involving the right ACA and partial bilateral MCA territories. A, ADC map shows areas of restricted diffusion in the right ACA, M1, M2, M4, and M5 territories as well as the left M2, M4, and M5 territories. B, mIP-SWI shows markedly hyperintense sulcal veins in the right $A C A, M 1, M 2, M 4$, and $M 5$ territories and hypointense sulcal veins in the left $M 1, M 2, M 4$, and $M 5$ territories. $C$ and $D$, Follow-up axial CT image 2 days after AIS shows stroke evolution in the right ACA, M1, M2, M4, and M5 as well as in the left M2, M4, and M5 territories. In addition, there is increasing mass effect with effacement of both frontal horns of the lateral ventricles, the third and fourth ventricles, and prepontine cistern, compatible with malignant edema.

Table 3: SWI hyperintense venous signal does not predict the occurrence of postischemic malignant edema in 24 children with AIS $^{\mathrm{a}}$

\begin{tabular}{lccc}
\hline $\begin{array}{c}\text { Venous Signal } \\
\text { Intensity on SWI }\end{array}$ & $\begin{array}{c}\text { No Malignant } \\
\text { Edema }\end{array}$ & $\begin{array}{c}\text { Malignant } \\
\text { Edema }\end{array}$ & Total \\
\hline Hypointense (only) & 14 & 0 & 14 \\
Hyperintense & 8 & 2 & 10 \\
Total & 22 & 2 & 24 \\
\hline
\end{tabular}

${ }^{\text {a Pearson }} \chi^{2}$ test $=0.07$

\section{DISCUSSION}

SWI has been increasingly shown to be a useful non-contrastenhanced imaging sequence in the evaluation of AIS. ${ }^{9-13,15}$ SWI may do the following: 1) detect hemorrhagic components within infarcted tissue with higher sensitivity than other MR imaging sequences or imaging modalities, ${ }^{16} 2$ ) demonstrate hypointense signals in the veins draining hypoperfused areas and evaluate the ischemic penumbra by focusing on the venous drainage, ${ }^{9-12} 3$ ) show hyperintense signal in the veins draining regions of hyperperfusion or luxury perfusion indicating an increased risk of developing postischemic malignant edema, ${ }^{13} 4$ ) detect acute occlusive arterial thromboemboli, ${ }^{17,18} 5$ ) quantify microhemorrhages and predict hemorrhagic transformation before thrombolytic 
therapy is initiated, ${ }^{19}$ and 6) detect early hemorrhagic complications after intra-arterial thrombolysis. ${ }^{17}$ Currently, most studies focus on adults, and literature on the role of SWI in PAIS is scant, based mostly on case reports or small case series. This is the largest study on SWI in PAIS to date.

Ischemic penumbra is characterized by hypoperfused brain tissue with the potential for functional recovery without morphologic damage. ${ }^{20}$ This is possible if local blood flow can be reestablished at a sufficient level within a certain time interval. The identification of the ischemic penumbra is important because it represents tissue that could potentially be salvaged with the use of thrombolytic therapy. Although the benefit of thrombolytic therapy in PAIS has yet to be demonstrated in prospective clinical trials, retrospective studies show that at least a subset of children with AIS may potentially benefit from it. ${ }^{21,22}$ Several tertiary pediatric centers have developed standardized systematic strategies to diagnose and treat PAIS, for participation in the ongoing Thrombolysis in Pediatric Stroke trial. ${ }^{21,22}$ Ischemic penumbra can be depicted as a mismatch between reduced perfusion and normal diffusion by combining PWI and DWI/DTI. ${ }^{23}$ In children, however, the routine application of PWI outside research protocols and tertiary care centers is still limited. DSC-PWI requires a rapid bolus injection of intravenous paramagnetic contrast agents that may delay acute antithrombotic therapy. ASL is a non-contrast-enhanced PWI method capable of measuring tissue perfusion by using blood as an endogenous "contrast" agent. ASL is not routinely performed in the acute setting because of the low SNR and limited spatial resolution. In addition, changes in signal intensity on ASL may be determined by factors other than reduced flow or ischemia, and knowledge of ASL-related artifacts is crucial for accurate interpretation.

The relationship between $\mathrm{CBF}$ and the oxygen extraction fraction (OEF) has been shown in both animal stroke models and humans by using PET, and 4 patterns have been observed in focal brain ischemia. ${ }^{24,25}$ In the first pattern, an increase in CBV was observed to maintain $\mathrm{CBF}$ in response to physiologic conditions or demands (autoregulation). The second pattern is an increase of OEF to maintain a stable cerebral metabolic rate of oxygen $\left(\mathrm{CMRO}_{2}\right)$ in response to a reduction of CBF. The third pattern is characterized by an increase of OEF in brain regions with reduction of both $\mathrm{CBF}$ and $\mathrm{CMRO}_{2}$ and represents ischemic penumbra. The fourth pattern represents the infarct core and is characterized by very low $\mathrm{CBF}$ and $\mathrm{CMRO}_{2}$ with poor OEF. SWI accentuates the magnetic susceptibility differences between deoxygenated hemoglobin in the vessels and adjacent oxygenated tissues and is an ideal MR imaging sequence to depict changes in OEF noninvasively. In a retrospective study including 15 adult patients with nonlacunar ischemic stroke, Kao et $\mathrm{al}^{10}$ showed that both SWIDWI and PWI (MTT)-DWI mismatches were significantly associated with a higher incidence of infarct progression and had a similar ability to predict stroke evolution. In addition, they showed that semiquantitative evaluation of SWI patterns and MTT values correlated best in the MCA territories. The authors concluded that SWI is an alternative to PWI to assess ischemic penumbra and predict stroke evolution. In children, the role of SWI in depicting the ischemic penumbra was previously shown only in a small case series and a few case reports. ${ }^{9,12,26}$ In our present study, we found a mismatch between vascular territories with SWI-hypointense venous signal and restricted diffusion in 6 children. In 5 of them, stroke progression was observed at followup. By contrast, stroke progression on follow-up was found in only 2 of 8 children without SWI-DTI mismatch. Our results show that a mismatch between vascular territories with SWI-hypointense venous signal and restricted diffusion is significantly associated with infarct progression in PAIS.

Postischemic malignant edema is a life-threatening early complication of PAIS and is defined as a space-occupying AIS with severe brain swelling and mass effect, which may cause transtentorial herniation and brain death. ${ }^{27,28}$ Impaired autoregulation in patients with stroke has been shown to lead to a rise in regional CBF followed by increased extravasation of fluid into the brain tissue and edema. ${ }^{29}$ This process is enhanced by disruption of the blood-brain barrier. ${ }^{30}$ The increase in CBF results in reduction of $\mathrm{OEF}$ and subsequently in a higher concentration of oxygenated hemoglobin and SWI-hyperintense venous signal. In 1 child with AIS, SWI was shown to determine regions of hyperperfusion or luxury perfusion, which contributed to an increased risk of developing malignant edema. ${ }^{13}$ To test our second hypothesis, we compared the occurrence of postischemic malignant edema in children with and without hyperintense venous signal on acute SWI. Our results show that hyperintense venous signal on acute SWI is not significantly associated with the development of malignant edema in PAIS.

Although this is the largest cohort of PAIS studied by SWI published to date, we are aware of several limitations of our study: the retrospective nature of the study resulting in the absence of follow-up neuroimaging studies in 10 children; the availability of ASL data in only 7 children; the semiquantitative analysis of the images, which is investigator-dependent though performed by consensus; differences in section thickness between ADC maps and mIP-SWI possibly having caused partial misregistration of some images; and no attempt to adjust for potential confounders with multiple logistic regression due to the rather small number of patients.

\section{CONCLUSIONS}

Our study shows that a mismatch between vascular territories with SWI-hypointense venous signal and restricted diffusion predicts infarct progression in PAIS, while hyperintense venous signal on acute SWI does not predict the development of malignant edema. SWI provides valuable information in PAIS and may guide early treatment options to prevent infarct progression and should be added to the neuroimaging diagnostic protocol of PAIS. Prospective studies with a larger cohort of children, a standardized follow-up interval, and ASL as part of the acute neuroimaging protocol are needed to further validate the value of SWI in PAIS.

\section{REFERENCES}

1. Amlie-Lefond C, Sébire G, Fullerton HJ. Recent developments in childhood arterial ischaemic stroke. Lancet Neurol 2008;7:425-35

2. deVeber GA, MacGregor D, Curtis R, et al. Neurologic outcome in survivors of childhood arterial ischemic stroke and sinovenous thrombosis. J Child Neurol 2000;15:316-24

AJNR Am J Neuroradiol 36:783-88 Apr 2015 www.ajnr.org 
3. Ganesan V, Hogan A, Shack N, et al. Outcome after ischaemic stroke in childhood. Dev Med Child Neurol 2000;42:455-61

4. Monagle P, Chalmers E, Chan A, et al. Antithrombotic therapy in neonates and children: American College of Chest Physicians Evidence-Based Clinical Practice Guidelines (8th Edition). Chest 2008; 133(6 suppl):887S-968S

5. Roach ES, Golomb MR, Adams R, et al. Management of stroke in infants and children: a scientific statement from a Special Writing Group of the American Heart Association Stroke Council and the Council on Cardiovascular Disease in the Young. Stroke 2008;39: 2644-91

6. Rafay MF, Pontigon AM, Chiang J, et al. Delay to diagnosis in acute pediatric arterial ischemic stroke. Stroke 2009;40:58-64

7. Gadian DG, Calamante F, Kirkham FJ, et al. Diffusion and perfusion magnetic resonance imaging in childhood stroke. J Child Neurol 2000;15:279-83

8. Bosemani T, Poretti A, Huisman TA. Susceptibility-weighted imaging in pediatric neuroimaging. J Magn Reson Imaging 2014;40: $530-44$

9. Chalian M, Tekes A, Meoded A, et al. Susceptibility-weighted imaging $(\mathrm{SWI})$ : a potential non-invasive imaging tool for characterizing ischemic brain injury? J Neuroradiol 2011;38:187-90

10. Kao HW, Tsai FY, Hasso AN. Predicting stroke evolution: comparison of susceptibility-weighted MR imaging with MR perfusion. Eur Radiol 2012;22:1397-403

11. Kesavadas C, Thomas B, Pendharakar H, et al. Susceptibility weighted imaging: does it give information similar to perfusion weighted imaging in acute stroke? J Neurol 2011;258:932-34

12. Meoded A, Poretti A, Benson JE, et al. Evaluation of the ischemic penumbra focusing on the venous drainage: the role of susceptibility weighted imaging (SWI) in pediatric ischemic cerebral stroke. J Neuroradiol 2014;41:108-16

13. Bosemani T, Poretti A, Orman G, et al. Pediatric cerebral stroke: susceptibility-weighted imaging may predict post-ischemic malignant edema. Neuroradiol J 2013;26:579-83

14. Pexman JH, Barber PA, Hill MD, et al. Use of the Alberta Stroke Program Early CT Score (ASPECTS) for assessing CT scans in patients with acute stroke. AJNR Am J Neuroradiol 2001;22:1534-42

15. Santhosh K, Kesavadas C, Thomas B, et al. Susceptibility weighted imaging: a new tool in magnetic resonance imaging of stroke. Clin Radiol 2009;64:74-83

16. Wycliffe ND, Choe J, Holshouser B, et al. Reliability in detection of hemorrhage in acute stroke by a new three-dimensional gradient recalled echo susceptibility-weighted imaging technique compared to computed tomography: a retrospective study. J Magn Reson Imaging 2004;20:372-77

17. Mittal S, Wu Z, Neelavalli J, et al. Susceptibility-weighted imaging: technical aspects and clinical applications, part 2. AJNR Am J Neuroradiol 2009;30:232-52

18. Verschuuren S, Poretti A, Buerki S, et al. Susceptibility-weighted imaging of the pediatric brain. AJR Am J Roentgenol 2012;198: W440-49

19. Akter M, Hirai T, Hiai Y, et al. Detection of hemorrhagic hypointense foci in the brain on susceptibility-weighted imaging clinical and phantom studies. Acad Radiol 2007;14:1011-19

20. Astrup J, Siesjö BK, Symon L. Thresholds in cerebral ischemia: the ischemic penumbra. Stroke 1981;12:723-25

21. Bernard TJ, Rivkin MJ, Scholz K, et al. Emergence of the primary pediatric stroke center: impact of the Thrombolysis in Pediatric Stroke Trial. Stroke 2014;45:2018-23

22. Marecos C, Gunny R, Robinson R, et al. Are children with acute arterial ischaemic stroke eligible for hyperacute thrombolysis? A retrospective audit from a tertiary UK centre. Dev Med Child Neurol 2015;57:181-86

23. Albers GW. Expanding the window for thrombolytic therapy in acute stroke. The potential role of acute MRI for patient selection. Stroke 1999;30:2230-37

24. Baron JC. Mapping the ischaemic penumbra with PET: implications for acute stroke treatment. Cerebrovasc Dis 1999;9:193-201

25. Baron JC, Rougemont D, Soussaline F, et al. Local interrelationships of cerebral oxygen consumption and glucose utilization in normal subjects and in ischemic stroke patients: a positron tomography study. J Cereb Blood Flow Metab 1984;4:140-49

26. Meoded A, Poretti A, Tekes A, et al. Clinical vignette: role of susceptibility-weighted imaging in predicting stroke evolution. Neurographics 2013;3:159-63

27. Ramaswamy V, Mehta V, Bauman M, et al. Decompressive hemicraniectomy in children with severe ischemic stroke and lifethreatening cerebral edema. J Child Neurol 2008;23:889-94

28. Rahme R, Jimenez L, Bashir U, et al. Malignant MCA territory infarction in the pediatric population: subgroup analysis of the Greater Cincinnati/Northern Kentucky Stroke Study. Childs Nerv Syst 2013;29:99-103

29. Dohmen C, Bosche B, Graf R, et al. Identification and clinical impact of impaired cerebrovascular autoregulation in patients with malignant middle cerebral artery infarction. Stroke 2007;38:56-61

30. Treadwell SD, Thanvi B. Malignant middle cerebral artery (MCA) infarction: pathophysiology, diagnosis and management. Postgrad Med J 2010;86:235-42 